\title{
Gold-catalyzed Dearomatization Reactions
}

\author{
Juzeng An and Marco Bandini*
}

\begin{abstract}
The advent of homogenous gold catalysis has revolutionized the way of approaching organic synthesis providing new and desirable solutions for challenging chemical manipulations of unactivated unsaturated hydrocarbons. In the realm of aromatic compounds, dearomatization protocols have been emerging as valuable synthetic tools to densely functionalized polycyclic 3D-molecular scaffolds, starting from readily available 2D-aromatic congeners. Gold catalysis contributed substantially in expanding this scenario enabling the condensation of numerous electron-rich arenes with hydrocarbons, resulting in the partial or total dearomatization of the aryl fragment. An overview of the most recent findings in the field, organized by type of electrophilic partners, is hereby presented.
\end{abstract}

Keywords: Catalysis · Dearomatization · Enantioselection · Gold · Selectivity
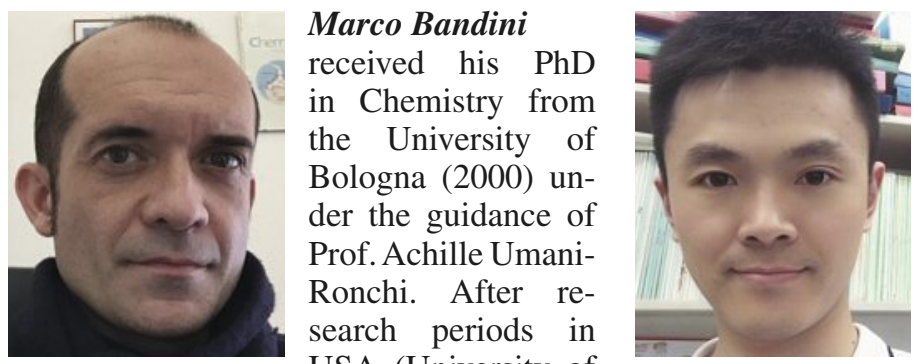

Juzeng An

was born and raised in Shandong, China. He obtained his BSc from Dezhou University in 2013. He then obtained his master degree under the supervision of Prof. GuoHua Liu in USA (University of North Carolina at Chapel Hill) and UK (University of York), he became Assistant Professor (2001) and Associate Professor (2012) at the Department of Chemistry 'G. Ciamcian' - Alma Mater Studiorum University of Bologna. His research interests cover the development of new organometallic catalytic systems ( $\mathrm{Au}(\mathrm{I})$ and 3d-TMs) for organic transformations. His personal track record comprises 118 publications, more than 7000 citations and 45 h-index.

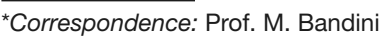
Dipartimento di Chimica "G. Ciamician" Alma Mater Studiorum - Università di Bologna

Via Selmi 2, Bologna, Italy

E-mail: marco.bandini@unibo.it forming the electrophilic activation of unsaturated hydrocarbons makes the siteselective manipulation of aromatic compounds with $\pi$-systems feasible under extremely mild conditions. Enantioselection is also accessible by means of chiral gold complexes. However, being in its infancy, many limitations can still be found. Among the others it is worthy of mention that while gold catalysis found satisfactory results in the dearomatization of electron-rich hetero-compounds (i.e. indoles, naphthols and phenols), electron-deficient as well as electron-neutral arenes still suffer from poor reactivity in the dearomative process. Additionally, intermolecular methodologies are far less investigated than intramolecular ones and developments in these directions are expected in the near future.

In the present mini-review, the most recent findings regarding the use of gold complexes in dearomative protocols, have been collected and organized based on the type of $\pi$-system employed. There is no intent to provide a comprehensive and exhaustive picture of the field; on the contrary, we wish to provide a useful guideline to practitioners emphasizing scopes, limitations and possible perspectives of this emerging research field. celivity. In this realm, catalysis is the ultimate tool[2] and homogenous gold(I) catalysis $^{[3]}$ contributes substantially in discovering, developing and consolidating new opportunities for the direct realization of densely functionalized polycyclic compounds. The main strategy for the gold-assisted dearomatization of aromatic compounds relies on the condensation of electron-rich arenes/ heteroarenes with $\pi$-systems (i.e. alkynes, allenes, alkenes) via inter- as well as intramolecular processes (Fig. 1). ${ }^{[4]}$ The unique aptitude of gold(I) species in per-

\section{Results and Discussion}

\subsection{Alkynes as Electrophilic Partners}

Alkynes are probably the most used unsaturated hydrocarbons in combination with homogenous gold catalysis. ${ }^{[5]}$ Therefore, it is not surprising to record that the highest number of gold-catalyzed dearomative examples involves the condensation of substituted electron-rich 
arenes with activated as well as unactivated triple bonds. These protocols commonly describe intramolecular condensations with properly designed molecular scaffolds that, upon cyclization result in 5- or 6-membered polycyclic structures. In terms of arenes, phenols and indoles are the main players and the use of substituted alkynes proved competent in triggering cascade reactions with applications in the synthesis of naturally occurring compounds. ${ }^{6]}$ It is worthy to mention that no intermolecular variants of alkyne-assisted dearomative protocols by means of gold catalysis have been described up to now.

\subsubsection{Dearomatization of Indoles}

The pioneering work by Echavarren and coworkers ${ }^{[7]}$ in 2007 paved the way for many research teams that addressed their efforts in realizing structurally elaborated compounds via gold-catalyzed activation of alkynes. ${ }^{[8]}$

In 2015, the Toste group ${ }^{[9]}$ reported the synthesis of enantiomerically enriched cyclopenta $[b]$ indoles 2 (ee up to $97 \%$ ) by means of enantioselective Rautenstrauchlike rearrangement of $\mathrm{C}(3)$-substituted indoles (Scheme 1a).

Later on, the site-selective dearomatization of $\mathrm{N}$-propargyl-2-bromo$\beta$-tryptamines was described by Guinchard and coworkers in a diastereoselective protocol [up to 93:7]. ${ }^{[10 \mathrm{a}, \mathrm{c}]}$ The presence of the bromine atom at the $\mathrm{C}(2)$-position of the acyclic starting material played a double function: i) preventing the re-aromatization of the system; 2) enabling the formation of the corresponding oxo-indole via final treatment with TFA. Shortly after, the same team also documented on the enantioenriched synthesis of the $\mathrm{C}(2)$ spiro-indolines 4 via gold-catalyzed enantioselective desymmetrization of the $N, N$ dipropargyl tryptamine 3 (er up to $84: 16$, Scheme 1b). ${ }^{[10 b]}$

\subsubsection{Dearomatization of Phenols/ Naphthols}

The extension of the protocol to oxygenated compounds (i.e. phenols) was not long in coming. In particular, Hamada was the first to document the dearomatization of 4-substituted phenols in the presence of IPrAuNTf 2 (5 mol\%) and $\mathrm{MsOH}(100$ mol\%).[11a] Almost simultaneously, the intramolecular dearomatization of aryl alkynoate esters ( $\mathrm{PPh}_{3} \mathrm{AuCl} / \mathrm{AgOTf}, 5$ mol\%) was reported by Vadola and colleagues. ${ }^{[11 b, c]}$ It is worth mentioning that the latter approach was also very recently implemented in a di-functionalization of the triple bond by combining gold catalysis and visible-light photoredox catalysis. [11d]

Subsequently, the combination of $\mathrm{PPh}_{3} \mathrm{AuCl}$ and $\mathrm{AgOMs}$ (both $5 \mathrm{~mol} \%$ ) was successfully adopted in the dearo-

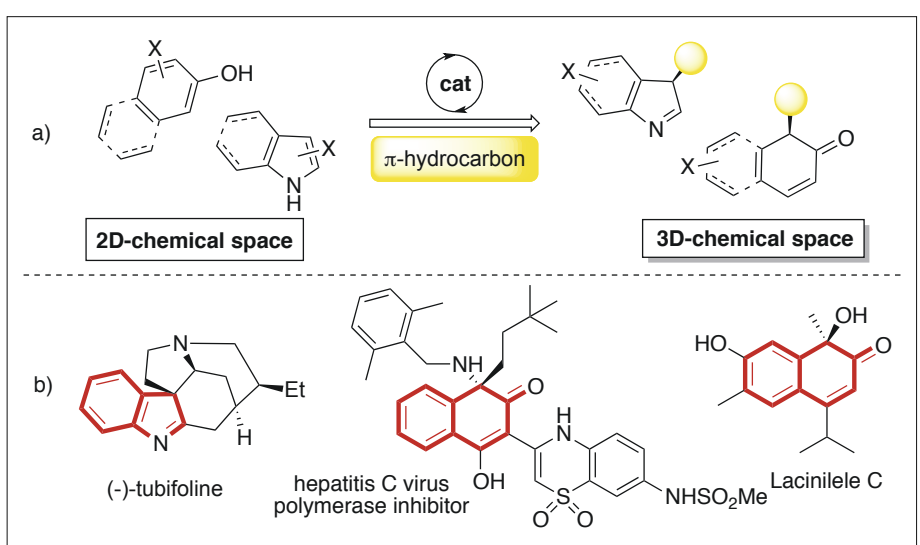

Fig. 1. a) From 2D- to 3D-chemical space: gold-catalyzed dearomatization reactions. b). Biologically relevant compounds featuring dearomatized indolines and 2-naphthyl units.

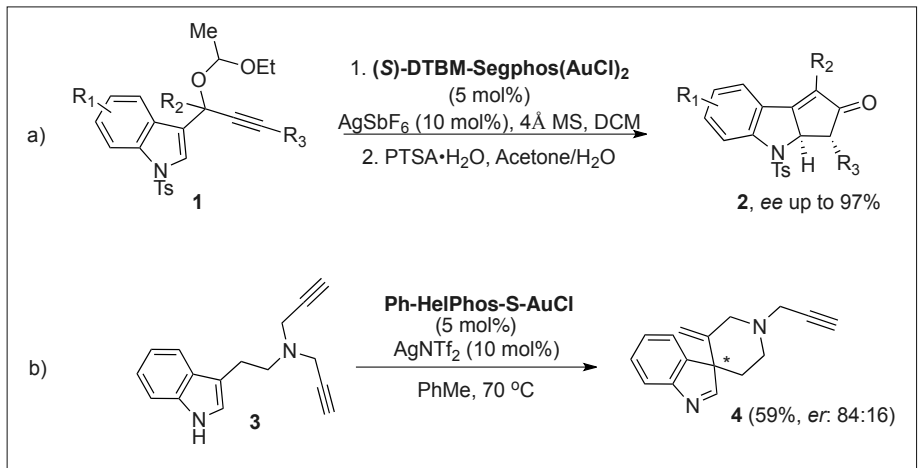

Scheme 1. a) Examples of gold catalyzed dearomatization of indoles via alkyne rearrangement; b) hydroindolination of alkynes.

matization/ipso-cyclization of 4-substituted 1-naphthols 5 by You and Zhang.[12] Preliminary insights into the realization of an enantioselective variant were also provided by means of chiral counterion methodology (ee up to $90 \%$, Scheme 2 a). Excellent functional group tolerance and high yields (up to 99\%) were obtained under mild reaction conditions.

Our group has recently joined the field by documenting a combined experimental/computational gold catalyzed (JohnPhosAu(ACN)SbF, 2.5 mol\%) [3,3]-sigmatropic rearrangement/ dearomative coupling/allenyl-trapping sequence, leading to regio- and stereochemically defined naphthalen-2(1H)ones 8 in high yields (up to $99 \%$, Scheme 2b). ${ }^{[13 a]}$ Mild reaction conditions (rt, reagent grade solvent, no moisture restrictions) and short reaction time (mins) enforced the synthetic appeal of the protocol. Simultaneously, the Gagné group ${ }^{[13 \mathrm{~b}]}$ reported a similar dearomative approach of 2-naphthols by subjecting aryloxy ethers comprising allylic, propargylic as well as allenylic side-arms to $\mathrm{PPh}_{3} \mathrm{AuNTf}_{2}$ catalysis $(5-10 \mathrm{~mol} \%)$. Interestingly, the dearomatization of the 2,4,6-trimethylphenol was also realized by condensing the transient $\mathrm{C}(2)$-allylated intermediate with a dienophile trap.

Conventional dearomatization/ipsocyclization of naphthols leads to unsaturated ketones that has rarely been subjected to subsequent chemical events. However, van der Eycken and colleagues have proposed an interesting cascade se- quence based on the use of properly designed substituted 1-naphthols ${ }^{[14 a]}$ and phenols. ${ }^{[14 b]}$ For a rapid generation of the acyclic precursors (9), the team took advantage of their expertise in the Ugi condensation, running a four-component variant encompassing electron-rich aromatic arenes such as naphthols, phenols and indoles. The use of $\mathrm{PPh}_{3} \mathrm{AuOTf}$ (10 mol\%) or IPrAuOTf (10 mol\%, Scheme 2c) triggered the cascade protocol via selective activation of the $\mathrm{C}-\mathrm{C}$ triple bond. A range of polyheterocyclic scaffolds $\mathbf{1 0}$ was obtained in very high yield and absolute diastereoselectivity.

\subsection{Allenamides/Allenoates and Ynamides as Electrophilic Partners}

Electronically modified $\pi$-systems such as allenamides (i.e. $\mathrm{N}$-allenyl amides), allenoates and ynamides have received great attention by the chemical community due to their ready availability, easy chemical decoration and property fine-tuning by means of metal as well as metal-free catalysis. ${ }^{[15]}$ In this context, our group documented the $[\mathrm{Au}(\mathrm{I})]$-catalyzed intermolecular condensation of $\mathrm{N}$-allenyl amides 12 with $\mathrm{C}(2,3)$-disubstituted indoles to give $\mathrm{C}(3)$-allylated indolines. ${ }^{[16 a]}$ The regioselectivity of the protocol was entirely governed by the metal counterion, with TFA (i.e. trifluoroacetate) being far superior with respect to less coordinating ones (Scheme 3a). ${ }^{[16 b]}$ The enantioselective variant of this dearomative process was presented by Chen in the presence of chiral C(1)-symmetric phosphoramidite 
gold complexes. A range of pyrroloindolines was realized in excellent yields and very high enantiomeric excesses (ee up to $97 \%$ ).[17] Subsequently, we also realized the enantioselective synthesis of $\mathrm{C}(2,3)$-cyclobutane-fused indolenines $\mathbf{1 4}$ with a similar approach by introducing an electron-withdrawing group at the $N(1)$ position of the indole that favored the formal [2+2]-cycloaddition event (Scheme 3b). ${ }^{[18]}$ With an analogous approach but through a [5+2]-cycloaddition, very recently Yoo and coworkers documented the $\mathrm{AuCl}(5 \mathrm{~mol} \%)$ dearomatization of quinolinium zwitterions with allenamides to provide 1,4-diazepines in high yields. [19]

Very recently, ynamides have also found application in the IPrAuNTf 2 catalyzed ( $3 \mathrm{~mol} \%)$ oxidative intramolecular annulation of $N$-alkylated tryptamines $\mathbf{1 5}$ with pyridine $N$-oxide (16). ${ }^{[20]}$ The methodology exploits the unique reactivity of gold-carbene intermediates that can be conveniently generated in situ by avoiding the use of hazardous diazo compounds.[21] A library of tetracyclic-fused spiroindolines $\mathbf{1 7}$ is obtained in very high yield and diastereoselection (Scheme 3c).

Allenoates are $\pi$-systems featuring complementary electronic properties with respect to $N$-allenyl amides and ynamides (electron-poor vs electron-rich allenyl groups). Despite that, $\mathrm{PPh}_{3} \mathrm{AuCl} / \mathrm{AgOTf}$ (5 mol\%) promotes the ipso-dearomatization of phenols that tether allenoate groups. The methodology proved competent for the preparation of densely functionalized di-spirocyclohexadienones. ${ }^{[22]}$

\subsection{Diazo- as Electrophilic Partners}

The metal-assisted transfer of carbenes from diazo compounds is a current hot topic in organic synthesis and gold catalysis has been recently applied to the selective dearomatization of heteroarenes (i.e. indoles and benzofurans). ${ }^{[23]}$ In particular, Sun and coworkers documented the condensation of diazoesters $\mathbf{1 8}$ with nitrogenprotected indoles followed by treatment with silica gel and air (IPrAu( $\mathrm{PhCN}) \mathrm{BAr}_{\mathrm{F}}$, $5 \mathrm{~mol} \%)$.[24a] Under these conditions, the (Z)-3-methyleneindoline intermediate 19 underwent a metal-free Schenck ene-type aerobic oxidation to form oxoindoles $\mathbf{2 0}$ in a high diastereoselective manner (Scheme 4). Similar reactivity but with reverse regioselectivity $(\mathrm{C}(2)$ vs $\mathrm{C}(3))$ was also documented by the same team in the presence of benzofurans. ${ }^{[24 \mathrm{~b}]}$

\section{Conclusions}

In summary, the direct and selective creation of chemical complexity/diversity from cheap and readily available starting materials is a 'holy-grail' of modern
Scheme 2. Synthesis of densely functionalized naphthalen-2(1H)-ones/ cyclohexanones via intramolecular gold(I)assisted alkyne trapping by phenols and naphthols. b)

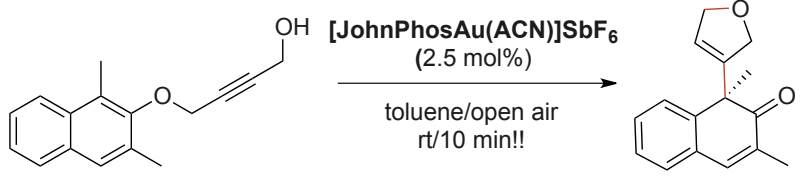

7

(+/-)-8 (97\%)

c)

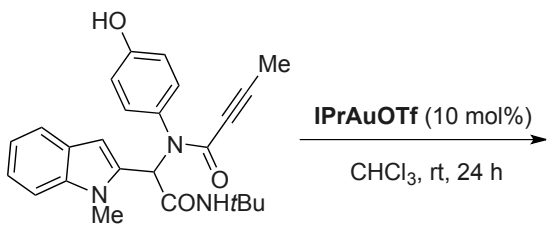

$(+/-)-9$

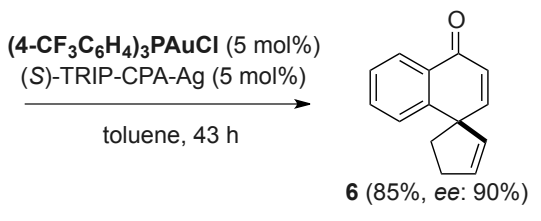

$6(85 \%$, ee: $90 \%)$

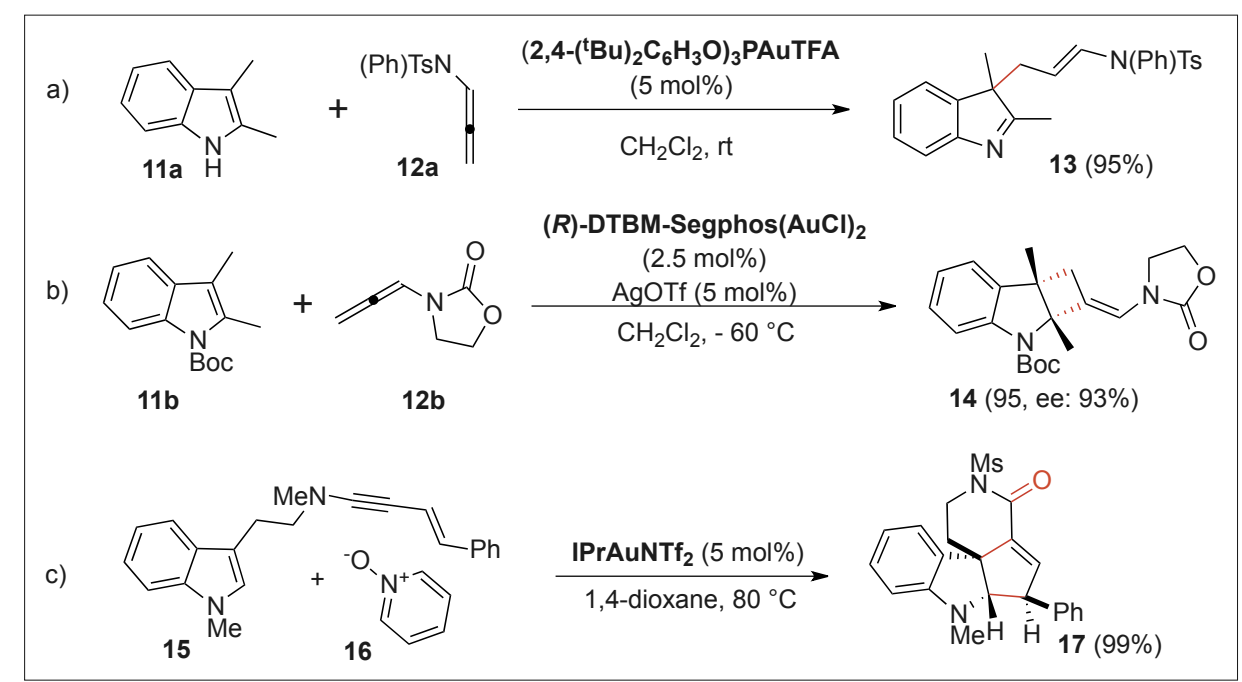

Scheme 3. Electron-rich allenes (i.e. $\mathrm{N}$-allenyl amides and ynamides) as valuable dearomatizing agents of indoles.

Scheme. 4. Synthesis of C(3)-functionalized oxoindoles via gold catalyzed condensation of diazo compounds with indoles followed by Schenck ene-type aerobic oxidation.

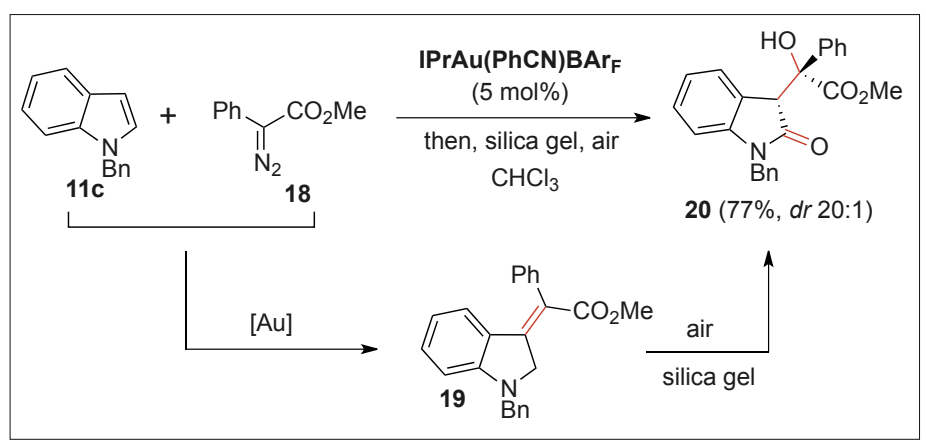

organic synthesis. The combination of homogenous gold catalysis and dearomative processes offers a unique opportunity to accomplish the target. Although being in its early stage developments, the goldcatalyzed dearomatization of electron-rich arenes is at the forefront in terms of synthesis of biologically relevant compounds and stereochemistry control. The undoubted scientific interest for this synthetic methodology, accompanied by some current important limitations such as adoptable arene and reaction-design suggest that further developments in the area should be considered a certitude.

\section{Acknowledgements}

JA is grateful to CSC programme No. 201608310110. University of Bologna is acknowledged for financial support. 
[1] a) A. R. Pape, K. P. Kaliappan, E. P. Kündig, Chem. Rev. 2000, 100, 2917; b) S. P. Roche, J. A. Porco, Angew. Chem. Int. Ed. 2011, 50, 4068.

[2] a) C.-X. Zhuo, W. Zhang, S.-L. You, Angew. Chem. Int. Ed. 2012, 51, 12662; b) C. Zheng, S.-L. You, Chem. 2016, 1, 830; c) 'Asymmetric Dearomatization Reactions' Ed. S.-L. You, Wiley-VCH, 2016; d) W.-T. Wu, L. Zhang, S. L. You, Chem. Soc. Rev. 2016, 45, 1570; e) X W. Liang, C. Zheng, S.-L. You, Chem. Eur. J. 2016, 22, 11918; f) S. Park, S. Chang, Angew. Chem. Int. Ed. 2017, 56, 7720.

[3] M. Bandini, Chem. Soc. Rev. 2011, 40, 1358.

[4] For a very recent review article on the topic see: W.-T. Wu, L. Zhang, S.-L. You, Acta Chim. Sinica 2017, 75, 419.

[5] a) R. Dorel, A. M. Echavarren, Chem. Rev 2015, 115, 9028; b) W. Debrouwer, T. S. A Heugebaert, B. I. Roman, C. V. Stevens, $A d v$ Synth. Catal. 2015, 357, 2975.

[6] For general reviews and exemplificative articles see: a) A. S. K. Hashmi, M. Rudolph, Chem Soc. Rev. 2008, 37, 1766; b) Y. Y. Wang, Z. Y. Cao, J. Zhou, 'Gold Catalysis in the Synthesis of Natural Products: Heterocycle Construction via Direct C-X-Bond-Forming Reactions', in 'Au-Catalyzed Synthesis and Functionalization of Heterocycles', Ed. M. Bandini, Topics in Heterocyclic Chemistry, vol 46., 2016, Springer; c) P. Y. Toullec, V. Michelet, J. Isr Chem. DOI: 10.1002/ijch.201800002; See also d) M. S. Kirillova, M. E. Muratore, R. Dorel, A M. Echavarren, J. Am. Chem. Soc. 2016, 138 , 3671; e) D. Pflästerer, A. S. K. Hashmi, Chem. Soc. Rev. 2016, 45, 1331.

[7] C. Ferrer, C. H. M. Amijs, A. M. Echavarren, Chem. Eur. J. 2007, 13, 1358.
[8] a) Y. Liu, W. Xu and X. Wang, Org. Lett. 2010 , 12, 1448; b) G. Cera, P. Crispino, M. Monari, M. Bandini, Chem. Commun. 2011, 47, 7803; c) G. Cera, M. Chiarucci, A. Mazzanti, M. Mancinelli, M Bandini, Org. Lett. 2012, 14 1350 .

[9] W. Zi, H. Wu, F. D. Toste, J. Am. Chem. Soc. 2015, 137, 3225 .

[10] a) V. Magn, F. Blanchard, A. Marinetti, A. Voituriez, X. Guinchard, Adv. Synth. Catal. 2016, 358, 3355; b) V. Magné, A. Marinetti, V. Gandon, A. Voituriez, X. Guinchard, $A d v$. Synth. Catal. 2017, 359, 4036; c) V. Magné, P. Retailleau, A. Marinetti, A. Voituriez, X. Guinchard, Molbank, 2018, M985.

[11] a) T. Nemoto, N. Matsuo, Y. Hamada, $A d v$. Synth. Catal. 2014, 356, 2417; b) M. D. Aparece, P. A. Vadola, Org. Lett. 2014, 16 , 6008 ; c) T. L. Vacala, P. R. Carlson, A. ArreolaHester, C. G. Williams, E. W. Makhoul, P. A. Vadola, J. Org. Chem. 2018, 83, 1493; d) A. H. Bansode, S. R. Shaikh, R. G. Gonnade, N. T. Patil, Chem. Commun. 2017, 53, 9081.

[12] W.-T. Wu, R.-Q. Xu, L. Zhang, S.-L. You, Chem. Sci. 2016, 7, 3427.

[13] a) J. An, A. Parodi, M. Monari, M. Castiñeira Reis, C. Silva Lopez, M. Bandini, Chem. Eur. J. 2017, 23, 17473; b) M. T. Peruzzi, S. J. Lee, M. R. Gagné, Org. Lett. 2017, 19, 6256.

[14] a) Y. He, Z. Li, G. Tian, L. Song, L. Van Meervelt, E. V. Van der Eycken, Chem. Commun. 2017, 53, 6413; b) Y. He, Z. Li, K. Robeyns, L. VanMeervelt, E. V. Van der Eycken, Angew. Chem. Int. Ed. 2018, 57, 272.

[15] a) B. Alcaide, P. Almendros, C. Aragoncillo, Chem. Soc. Rev. 2010, 39, 783; b) T. Lu, Z. Lu, Z.-X. Ma, Y. Zhang, R. P. Hsung, Chem. Rev. 2013, 130, 4862; c) E. Manoni, M. Bandini,
Eur. J. Org. Chem. 2016, 3135; d) G. Evano, N. Blanchard, G. Compain, A. Coste, C. S. Demmer, W. Gati, C. Guissart, J. Heimburger, N. Henry, K. Jouvin, G. Karthikeyan, A. Laouiti, M. Lecomte, A. Martin-Mingot, B. Métayer, B. Michelet, A. Nitelet, C. Theunissen, S. Thibaudeau, J. Wang, M. Zarca, C. Zhang, Chem. Lett. 2016, 45, 574.

[16] a) M. Jia, G. Cera, D. Perrotta, M. Monari, M. Bandini, Chem. Eur. J. 2014, 20, 9875; b) L. Rocchigiani, M. Jia, M. Bandini, A Macchioni, ACS Catal. 2015, 5, 3911.

[17] Z.-Q. Shen, X.-X. Li, J.-W. Shi, B.-L. Chen, Z Chen, Tetrahedron Lett. 2015, 56, 4080.

[18] a) M. Jia, M. Monari, Q.-Q. Yang, M. Bandini, Chem. Commun. 2015, 51, 2320; b) R. Ocello, A. De Nisi, M. Jia, Q.-Q. Yang, M. Monari, P. Giacinto, A. Bottoni, G. P. Miscione, M. Bandini, Chem. Eur. J. 2015, 21, 18445.

[19] N. De, C. E. Song, D. H. Ryu, E. J. Yoo, Chem. Commun. 2018, 54, 6911.

[20] M. Lin, L. Zhu, J. Xia, Y. Yu, J. Chen, Z. Mao, X. Huang, Adv. Synth. Catal. 2018, 360, 2280.

[21] a) Z. Zheng, Z. Wang, Y. Wang, L. Zhang, Chem. Soc. Rev. 2016, 45, 4448; b) R.J. Harris, R. A. Widenhoefer, Chem. Soc. Rev. 2016, 45 , 4533.

[22] H. Wang, K. Wang, Y. Xiang, H. Jiang, X. Wan, N. Li, B. Tanga, Adv. Synth. Catal. 2018, 360 2352.

[23] D. Qian, J. Zhang, Chem. Soc. Rev. 2015, 44, 677.

[24] a) K. Liu, G. Xu, J. Sun, Chem. Sci. 2018, 9 , 634; b) G. Xu, K. Liu, J. Sun, Org. Lett. 2018 20,72 . 